\title{
Monotonicity properties and bounds involving the two-parameter generalized Grötzsch ring function
}

\author{
Guo-Jing Hai ${ }^{1}$ and Tie-Hong Zhao ${ }^{1 *}$
}

\section{"Correspondence:}

tiehong.zhao@hznu.edu.cn

'Department of Mathematics,

Hangzhou Normal University,

Hangzhou, China

\section{Springer}

\begin{abstract}
In the article, we present several new monotonicity properties and bounds involving the generalized Grötzsch ring functions $\mu_{a, b}$ in the theory of Ramanujan's generalized modular equation for $0<a, b<1$. Our results are the variants and extensions of some previously known results.
\end{abstract}

MSC: $33 \mathrm{C05} ; 33 \mathrm{E} 05$

Keywords: Gaussian hypergeometric function; Generalized Grötzsch ring function; Generalized elliptic integral; Monotonicity property

\section{Introduction}

Given $x \in(-1,1)$ and real numbers $a, b$, and $c$ with $c \neq 0,-1,-2, \ldots$, the Gaussian hypergeometric function $F(a, b ; c ; x)[1-18]$ is defined by

$$
F(a, b ; c ; x)={ }_{2} F_{1}(a, b ; c ; x)=\sum_{n=0}^{\infty} \frac{(a, n)(b, n)}{(c, n)} \frac{x^{n}}{n !}
$$

where $(a, 0)=1$ for $a \neq 0$ and $(a, n)=a(a+1)(a+2) \cdots(a+n-1)$ for $n=1,2, \ldots F(a, b ; c ; x)$ is said to be zero-balanced if $c=a+b$. If $x \rightarrow 1$, then the following asymptotic formulas

$$
\begin{cases}F(a, b ; c ; 1)=\frac{\Gamma(c) \Gamma(c-a-b)}{\Gamma(c-a) \Gamma(c-b)}, & a+b<c, \\ B(a, b) F(a, b ; c ; x)+\log (1-x)=R(a, b)+O((1-x) \log (1-x)), & a+b=c, \\ F(a, b ; c ; x)=(1-x)^{c-a-b} F(c-a, c-b ; c ; x), & a+b>c,\end{cases}
$$

can be found in the literature [19, Theorems 1.19 and 1.48], where $\Gamma(x)=\int_{0}^{\infty} t^{x-1} e^{-t} d t$ [20-26] and $B(p, q)=[\Gamma(p) \Gamma(q)] / \Gamma(p+q)$ [27-30] are respectively the classical Euler gamma and beta functions, and

$$
R(a, b)=-\psi(a)-\psi(b)-2 \gamma, \quad R\left(\frac{1}{2}, \frac{1}{2}\right)=\log 16, \quad \psi(x)=\frac{\Gamma^{\prime}(x)}{\Gamma(x)}
$$

(c) The Author(s) 2020. This article is licensed under a Creative Commons Attribution 4.0 International License, which permits use, sharing, adaptation, distribution and reproduction in any medium or format, as long as you give appropriate credit to the original author(s) and the source, provide a link to the Creative Commons licence, and indicate if changes were made. The images or other third party material in this article are included in the article's Creative Commons licence, unless indicated otherwise in a credit line to the material. If material is not included in the article's Creative Commons licence and your intended use is not permitted by statutory regulation or exceeds the permitted use, you will need to obtain permission directly from the copyright holder. To view a copy of this licence, visit http://creativecommons.org/licenses/by/4.0/. 
and

$$
\gamma=\lim _{n \rightarrow \infty}\left(\sum_{k=1}^{n} \frac{1}{k}-\log n\right)=0.5772156649 \ldots
$$

is the Euler-Mascheroni constant [31-33].

Ramanujan's generalized modular equation with order (or degree) $p>0$ is given by

$$
\frac{F\left(a, b ; c ; 1-s^{2}\right)}{F\left(a, b ; c ; s^{2}\right)}=p \frac{F\left(a, b ; c ; 1-r^{2}\right)}{F\left(a, b ; c ; r^{2}\right)}, \quad 0<r<1 .
$$

It is well known that equation (1.3) has a unique solution for $s$ if $a, b, c>0$ with $a+b \geq c$ [34, Lemma 4.5].

The two-parameter generalized Grötzsch ring function is defined by

$$
\mu_{a, b}(r)=\frac{B(a, b)}{2} \frac{F\left(a, b ;(a+b+1) / 2 ; 1-r^{2}\right)}{F\left(a, b ;(a+b+1) / 2 ; r^{2}\right)}, \quad r \in(0,1)
$$

if $a+b \geq 1$.

Our interest is to focus on $c=(a+b+1) / 2$, which makes the derivative formula of the two-parameter generalized Grötzsch ring function defined by (1.4) simpler.

Let $0<a, b<1$ with $a+b \geq 1$ and $r \in(0,1)$. Then the two-parameter generalized elliptic integrals of first and second kinds [34, (1.6)-(1.8)] are defined by

$$
\begin{aligned}
& \mathcal{K}=\mathcal{K}_{a, b}=\mathcal{K}_{a, b}(r)=\frac{B(a, b)}{2} F\left(a, b ; \frac{a+b+1}{2} ; r^{2}\right), \\
& \mathcal{E}=\mathcal{E}_{a, b}=\mathcal{E}_{a, b}(r)=\frac{B(a, b)}{2} F\left(a-1, b ; \frac{a+b+1}{2} ; r^{2}\right), \\
& \mathcal{K}^{\prime}=\mathcal{K}_{a, b}^{\prime}=\mathcal{K}_{a, b}\left(r^{\prime}\right), \quad \mathcal{E}^{\prime}=\mathcal{E}_{a, b}^{\prime}=\mathcal{E}_{a, b}\left(r^{\prime}\right),
\end{aligned}
$$

where and in what follows $r^{\prime}=\sqrt{1-r^{2}}$. Moreover, it follows from (1.2) that

$$
\begin{aligned}
& \mathcal{K}_{a, b}\left(0^{+}\right)=\mathcal{E}_{a, b}\left(0^{+}\right)=\frac{B(a, b)}{2} \\
& \mathcal{K}_{a, b}\left(1^{-}\right)=\infty, \quad \mathcal{E}_{a, b}\left(1^{-}\right)=\frac{B(a, b) B((a+b+1) / 2,(3-a-b) / 2)}{2 B((b-a+3) / 2,(a-b+1) / 2)} .
\end{aligned}
$$

In this paper, we study the two-parameter generalized Grötzsch ring function $\mu_{a, b}(r)$ for $a, b \in(0,1)$, as well as the related functions $\mathcal{K}_{a, b}, \mathcal{E}_{a, b}$, and

$$
m_{a, b}(r)=\frac{2}{B(a, b)} r^{\prime 2} \mathcal{K}_{a, b} \mathcal{K}_{a, b}^{\prime}, \quad r \in(0,1)
$$

The so-called Legendre $\mathcal{M}$-function introduced in [35] can be used to study the derivative of $m_{a, b}(r)$ and satisfies the formula

$$
\left[\frac{B(a, b)}{2}\right]^{2} \mathcal{M}\left(r^{2}\right)=\frac{a+b-1}{2} \mathcal{K K}^{\prime}+\frac{b-a+1}{2}\left(\mathcal{K} \mathcal{E}^{\prime}+\mathcal{K}^{\prime} \mathcal{E}-\mathcal{K} \mathcal{K}^{\prime}\right)
$$


for $r \in(0,1)$. Furthermore, $\mathcal{M}(r)$ can be rewritten as

$$
\mathcal{M}(r)=\frac{\Gamma((a+b+1) / 2)^{2}[r(1-r)]^{(1-a-b) / 2}}{\Gamma(a) \Gamma(b)},
$$

and $\mathcal{M}(r)$ becomes a constant if and only if $a+b=1$, in which case $\mathcal{M}\left(r^{2}\right)$ degenerates to be the generalized Legendre relation.

In the case of $a+b=1$, these functions coincide with the special functions $\mu_{a}(r), \mathcal{K}_{a}(r)$, $\mathcal{E}_{a}(r)$, and $m_{a}(r)$, respectively, which were studied in [36-49]. In particular, if $a=b=1 / 2$, then these functions reduce to the classical cases denoted by $\mu(r), \mathcal{K}(r), \mathcal{E}(r)$, and $m(r)$, which appeared frequently in the geometric function theory and number theory [50-69].

The main purpose of the article is to find the sub-regions of $\left\{(a, b) \in \mathbb{R}^{2} \mid 0<a, b<1, a+\right.$ $b>1\}$ such that certain quotient functions involving $\mu_{a, b}(r), \mathcal{K}_{a, b}(r), \mathcal{E}_{a, b}(r)$, and $m_{a, b}(r)$ are monotonic on their corresponding sub-regions. As a consequence, several new bounds for $\mu_{a, b}(r)$ and $m_{a, b}(r)$ are discovered, which are the variants and extensions of the results given in [42, Theorems 1.1 and 1.2] for the case of zero-balanced.

\section{Notations, formulas, and lemmas}

In order to prove our main results, we need several derivative formulas and lemmas, which we present in this section.

\subsection{Notations}

Throughout the article, we denote $B(a, b)$ by $B$ if no risk for confusion. Let

$$
\begin{aligned}
& D=\frac{B\left(\frac{a+b+1}{2}, \frac{a+b-1}{2}\right)}{2}, \\
& E=\frac{B(a, b) B\left(\frac{a+b+1}{2}, \frac{1-a-b}{2}\right)}{2 B\left(\frac{a-b+1}{2}, \frac{b-a+1}{2}\right)}, \\
& \kappa_{1}(a, b)=a+b+1-2 a b(4-a-b), \\
& \kappa_{2}(a, b)=3+7(a+b)+2(a-b)^{2}-6(a+b)^{3}-5(a+b)^{4}-(a+b)^{5} \\
& +8 a b\left[(a+b)^{2}+(a+b)^{3}-(a+b)+8 a b\right], \\
& \kappa_{3}(a, b)=5+7(a+b)-3(a+b)^{2}-7(a+b)^{3}-2(a+b)^{4} \\
& +4 a b\left[3(a+b)^{2}+4(a+b)-3\right] \\
& \kappa_{4}(a, b)=9+5(a+b)-9(a+b)^{2}-5(a+b)^{3}+16 a b(a+b), \\
& \kappa_{5}(a, b)=(a+b+1)^{2}-12 a b .
\end{aligned}
$$

For the convenience of readers, we also introduce three sub-regions $\Omega_{1}, \Omega_{2}$, and $\Omega_{3}$ of $\left\{(a, b) \in \mathbb{R}^{2} \mid 0<a, b<1\right\}$, which are illustrated in Fig. 1 .

$$
\begin{aligned}
& \Omega_{1}=\left\{(a, b) \mid 0<a, b<1, a+b>1, \kappa_{1}(a, b) \geq 0\right\}, \\
& \Omega_{2}=\left\{(a, b) \mid 0<a, b<1, a+b>1, \kappa_{2}(a, b) \leq 0, \kappa_{3}(a, b) \leq 0\right\}, \\
& \Omega_{3}=\left\{(a, b) \mid 0<a, b<1, a+b>1, \kappa_{5}(a, b) \geq 0\right\} .
\end{aligned}
$$






(a) $\Omega_{1}$

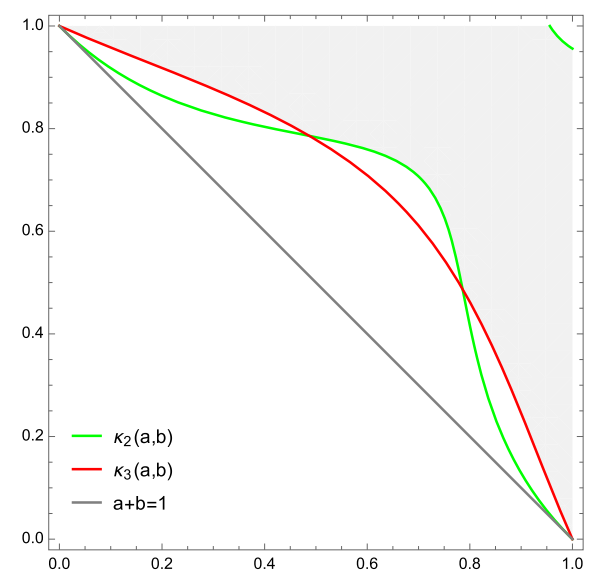

(b) $\Omega_{2}$

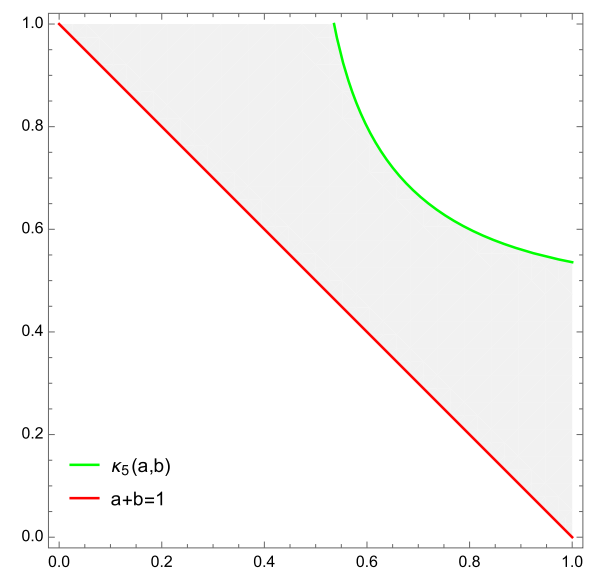

(c) $\Omega_{3}$

Figure 1 Visualized sub-regions $\Omega_{1}, \Omega_{2}$, and $\Omega_{3}$ of $\left\{(a, b) \in \mathbb{R}^{2} \mid 0<a, b<1\right\}$

\subsection{Formulas}

Let $r \in(0,1)$ and $0<a, b<1$ with $a+b>1$. Then the following derivative formulas

$$
\begin{aligned}
& \frac{d \mathcal{K}}{d r}=\frac{1}{r r^{\prime 2}}\left[2 b\left(\mathcal{E}-r^{\prime 2} \mathcal{K}\right)+(a+b-1)(\mathcal{K}-\mathcal{E})\right], \\
& \frac{d \mathcal{E}}{d r}=\frac{2(a-1)}{r}(\mathcal{K}-\mathcal{E}), \\
& \frac{d(\mathcal{K}-\mathcal{E})}{d r}=\frac{1}{r r^{\prime 2}}\left[2 b r^{2} \mathcal{K}-\left(a+b-1+2(1-a) r^{2}\right)(\mathcal{K}-\mathcal{E})\right], \\
& \frac{d\left(\mathcal{E}-r^{\prime 2} \mathcal{K}\right)}{d r}=\frac{1}{r}\left[2(1-b) r^{2} \mathcal{K}+(a+b-1)(\mathcal{K}-\mathcal{E})\right]
\end{aligned}
$$

can be found in [34, Theorem 4.15].

Note that Theorem 1.19(9) of [19] gives the derivative formula

$$
\frac{d \mu_{a, b}(r)}{d r}=-\frac{(a+b-1) B^{2} D}{4 r^{a+b} r^{\prime a+b+1} \mathcal{K}^{2}}
$$

for $\mu_{a, b}(r)$ if $d=c=(a+b+1) / 2$. 
From (1.7), (1.9), (1.10), and (2.1) we clearly see that

$$
\frac{d m_{a, b}(r)}{d r}=\frac{4}{B r}\left[\left((2 b-1) r^{2} \mathcal{K}-(b-a+1)(\mathcal{K}-\mathcal{E})\right) \mathcal{K}^{\prime}-\frac{(a+b-1) B D}{4\left(r r^{\prime}\right)^{a+b-1}}\right] .
$$

\subsection{Lemmas}

Lemma 2.1 ([70, Theorem 2.1]) Suppose that the power series $f(x)=\sum_{n=0}^{\infty} a_{n} x^{n}$ and $g(x)=$ $\sum_{n=0}^{\infty} b_{n} x^{n}$ have the radius of convergence $r>0$ with $b_{n}>0$ for all $n \in\{0,1,2, \ldots\}$. Let $h(x)=$ $f(x) / g(x)$ and $H_{f, g}=\left(f^{\prime} / g^{\prime}\right) g-f$. Then the following statements hold true:

(1) If the non-constant sequence $\left\{a_{n} / b_{n}\right\}_{n=0}^{\infty}$ is increasing (decreasing) for all $n \geq 0$, then $h(x)$ is strictly increasing (decreasing) on $(0, r)$;

(2) If there exists $n_{0}>0$ such that the non-constant sequence $\left\{a_{n} / b_{n}\right\}_{n=0}^{\infty}$ is increasing (decreasing) for $0 \leq n \leq n_{0}$ and decreasing (increasing) for $n \geq n_{0}$, then $h(x)$ is strictly increasing (decreasing) on $(0, r)$ if and only if $H_{f, g}\left(r^{-}\right) \geq(\leq) 0$. Moreover, if $H_{f, g}\left(r^{-}\right)<(>) 0$, then there exists $x_{0} \in(0, r)$ such that $h(x)$ is strictly increasing (decreasing) on $\left(0, x_{0}\right)$ and strictly decreasing (increasing) on $\left(x_{0}, r\right)$.

Lemma 2.2 ([19, Theorem 1.25]) Suppose that $-\infty<a<b<\infty, f, g:[a, b] \rightarrow \mathbb{R}$ are continuous on $[a, b]$ and differentiable on $(a, b)$, and $g^{\prime}(x) \neq 0$ on $(a, b)$. If $f^{\prime}(x) / g^{\prime}(x)$ is increasing (decreasing) on $(a, b)$, then so are the functions

$$
\frac{f(x)-f(a)}{g(x)-g(a)} \text { and } \frac{f(x)-f(b)}{g(x)-g(b)}
$$

If $f^{\prime}(x) / g^{\prime}(x)$ is strictly monotone, then the monotonicity in the conclusion is also strict.

Lemma 2.3 Let $0<a, b<1$ with $a+b>1$. Then the following assertions are valid:

(i) The function $(\mathcal{K}-\mathcal{E}) /\left(r^{2} \mathcal{K}\right)$ is strictly increasing from $(0,1)$ onto $(2 b /(a+b+1), 1)$;

(ii) The function $r^{\prime a+b-1} \mathcal{K}$ has positive Maclaurin coefficients and maps $(0,1)$ onto $(B / 2, D)$;

(iii) The function $r^{\prime} \mathcal{K}$ is strictly decreasing from $(0,1)$ onto $(0, B / 2)$ if $p \geq 4 a b /(a+b+1)$.

Proof Items (i) and (ii) follow directly from [34, Lemma 4.22]. We only need to prove item (iii).

It follows from (2.1) that

$$
\begin{aligned}
\frac{d\left(r^{\prime p} \mathcal{K}\right)}{d r} & =\frac{r^{\prime p-2}}{r}\left[(2 b-p) r^{2} \mathcal{K}-(b-a+1)(\mathcal{K}-\mathcal{E})\right] \\
& =(b-a+1) r r^{\prime p-2} \mathcal{K}\left[\frac{2 b-p}{b-a+1}-\frac{\mathcal{K}-\mathcal{E}}{r^{2} \mathcal{K}}\right] .
\end{aligned}
$$

Lemma 2.3(i) and (2.7) enable us to know that $r^{\prime p} \mathcal{K}$ is strictly decreasing on $(0,1)$ if $(2 b-$ $p) /(b-a+1) \leq 2 b /(a+b+1)$, that is, $p \geq 4 a b /(a+b+1)$.

Note that

$$
r^{\prime p} \mathcal{K}=\frac{B(a, b)}{2} r^{\prime p+1-a-b} F\left(\frac{b-a+1}{2}, \frac{a-b+1}{2} ; \frac{a+b+1}{2} ; r^{2}\right) .
$$

If $p \geq 4 a b /(a+b+1)$, then $p+1-a-b \geq(a-b+1)(b-a+1) /(a+b+1)>0$. This in conjunction with (1.2) and (2.8) gives $\lim _{r \rightarrow 1^{-}} r^{\prime p} \mathcal{K}=0$. 
In the following Lemma 2.4 we provide an asymptotic formula for $\mathcal{K}$ as $r \rightarrow 1$ in the case of $a+b>1$, which is the analog for the zero-balanced hypergeometric function (1.2).

Lemma 2.4 Let $0<a, b<1$ with $a+b>1$. Then one has

$$
\mathcal{K}(\sqrt{r})=D(1-r)^{(1-a-b) / 2}+E+o\left((1-r)^{\frac{a+b-1}{2}} \log (1-r)\right)
$$

as $r \rightarrow 1$.

Proof It follows from $F(a, b ;(a+b+1) / 2 ; r)$ is asymptotic to $2 D(1-r)^{(1-a-b) / 2} / B[19$, Theorem 1.19(5)] as $r \rightarrow 1$ for $a+b>1$ and the derivative formula

$$
\frac{d F(a, b ; c ; r)}{d r}=\frac{a b}{c} F(a+1, b+1 ; c+1 ; r)
$$

given in $[19,(1.16)]$ for the hypergeometric function together with (1.2), and L'Hôpital's rule that

$$
\begin{aligned}
\lim _{r \rightarrow 1^{-}} & \frac{\mathcal{K}(\sqrt{r})-D(1-r)^{(1-a-b) / 2}-E}{(1-r)^{(a+b-1) / 2} \log (1-r)} \\
\quad= & \lim _{r \rightarrow 1^{-}} \frac{B F\left(\frac{b-a+1}{2}, \frac{a-b+1}{2} ; \frac{a+b+1}{2} ; r\right)-2 D-2 E(1-r)^{(a+b-1) / 2}}{2(1-r)^{a+b-1} \log (1-r)} \\
\quad= & \lim _{r \rightarrow 1^{-}} \frac{\left[(b-a)^{2}-1\right] B F\left(\frac{b-a+3}{2}, \frac{a-b+3}{2} ; \frac{a+b+3}{2} ; r\right)-2\left[(a+b)^{2}-1\right] E(1-r)^{(a+b-3) / 2}}{4(a+b+1)(1-r)^{a+b-2}[(a+b-1) \log (1-r)+1]} \\
= & \lim _{r \rightarrow 1^{-}} \frac{\left[(b-a)^{2}-1\right] B F\left(a, b ; \frac{a+b+3}{2} ; r\right)-2\left[(a+b)^{2}-1\right] E}{4(a+b+1)(1-r)^{(a+b-1) / 2}[(a+b-1) \log (1-r)+1]} \\
\quad= & \lim _{r \rightarrow 1^{-}} \frac{a b\left[1-(b-a)^{2}\right] B(1-r)^{2-(a+b)} F\left(\frac{b-a+3}{2}, \frac{a-b+3}{2} ; \frac{a+b+5}{2} ; r\right)}{(a+b+3)\left[(a+b)^{2}-1\right][(a+b-1) \log (1-r)+3]} \\
\quad=0 . &
\end{aligned}
$$

This completes the proof.

Lemma 2.4 leads to Corollary 2.5 immediately.

Corollary 2.5 Let $0<a, b<1$ and $a+b>1$. Then

$$
D r^{1-a-b}+E-\mu_{a, b}(r) \rightarrow 0 \quad \text { and } \quad D r^{1-a-b}+E-m_{a, b}(r) \rightarrow 0
$$

as $r \rightarrow 0$.

Proof By replacing $r$ with $1-r^{2}$ in Lemma 2.4 , we clearly see that

$$
\mathcal{K}^{\prime}=D r^{1-a-b}+E+o\left(r^{a+b-1} \log r^{2}\right)
$$


By definition, it is easy to know that $(\mathcal{K}-B / 2) / r \rightarrow 0$ as $r \rightarrow 0$. This in conjunction with (2.10) and $a+b<2$ yields

$$
\begin{aligned}
& D r^{1-a-b}+E-\mu_{a, b}(r) \\
& \quad=\frac{B}{2 \mathcal{K}}\left(D r^{1-a-b}+E-\mathcal{K}^{\prime}\right)+\frac{1}{\mathcal{K}}(\mathcal{K}-B / 2)\left(D r^{1-a-b}+E\right) \rightarrow 0
\end{aligned}
$$

as $r \rightarrow 0$. The second asymptotic formula can be proved by similar arguments.

Lemma 2.6 Let $0<a, b<1$ with $a+b>1$. Then the following assertions are valid:

(i) If $\kappa_{1}(a, b) \geq 0$, then $\kappa_{5}(a, b)>0$ and $a+b<3 / 2$;

(ii) $\kappa_{4}(a, b)<\kappa_{3}(a, b)$;

(iii) If $\kappa_{5}(a, b) \geq 0$ and $a \leq b$, then $3-3 a-b>0$.

Proof (i) We only need to prove that it is not possible for $\kappa_{1}(a, b) \geq 0$ and $\kappa_{5}(a, b) \leq 0$. By calculations, the inequality $\kappa_{1}(a, b) \geq 0$ is equivalent to $0<a \leq 1 / 2$ and $1-a<b<1$ or $1 / 2<$ $a<1$ and $1-a<b \leq b_{1}(a)$, where $b_{1}(a)=\frac{1}{4 a}\left[-1+8 a-2 a^{2}-\sqrt{1-24 a+60 a^{2}-32 a^{3}+4 a^{4}}\right]$ and $\kappa_{5}(a, b) \leq 0$ is equivalent to $1 / 2<2(2-\sqrt{3})<a<1$ and $b_{2}(a, b) \leq b<1$, where $b_{2}(a)=$ $-1+5 a-2 \sqrt{3\left(2 a^{2}-a\right)}$.

It remains to show that $b_{2}(a)>b_{1}(a)$ for $2(2-\sqrt{3})<a<1$. A simple calculation leads to

$$
\begin{aligned}
b_{2}(a)-b_{1}(a)= & \frac{1}{4 a}\left[1-12 a+22 a^{2}+\sqrt{1-24 a+60 a^{2}-32 a^{3}+4 a^{4}}\right] \\
& -2 \sqrt{3 a(2 a-1)}>0
\end{aligned}
$$

if and only if

$$
\begin{aligned}
& \left(1-12 a+22 a^{2}+\sqrt{1-24 a+60 a^{2}-32 a^{3}+4 a^{4}}\right)^{2}-(8 a \sqrt{3 a(2 a-1)})^{2} \\
& =2\left[\left(22 a^{2}-12 a+1\right) \sqrt{1-24 a+60 a^{2}-32 a^{3}+4 a^{4}}\right. \\
& \left.\quad+1-24 a+124 a^{2}-184 a^{3}+52 a^{4}\right]>0,
\end{aligned}
$$

which is also equivalent to

$$
\begin{aligned}
\left(22 a^{2}-12 a+1\right)^{2}\left(1-24 a+60 a^{2}-32 a^{3}+4 a^{4}\right) \\
\quad-\left(1-24 a+124 a^{2}-184 a^{3}+52 a^{4}\right)^{2} \\
=64 a^{3}(a+1)(3-2 a)(2 a-1)\left(3 a^{2}-3 a+1\right)>0
\end{aligned}
$$

for $1 / 2<a<1$. On the other hand, as we know, $\kappa_{1}(a, b)$ can be thought of as a quadratic function of $b$ and the parabola opens up. It is easy to verify that $\kappa_{1}(a, 1-a)=2(1-3 a+$ $\left.3 a^{2}\right)>0$ and $\kappa_{1}(a, 1)=-(2-a)(2 a-1)<0$ for $1 / 2<a<1$. Combining this with $\kappa_{1}(a, 3 / 2-$ $a)=-5(1-a)(a-1 / 2)<0$ for $1 / 2<a<1$, we conclude that $3 / 2-a<b<1$ makes $\kappa_{1}(a, b)$ negative. This completes the first assertion.

(ii) Observe that $\kappa_{4}(a, b)-\kappa_{3}(a, b)=2(a+b+1)(a+b-1) \mathcal{Q}(b)$, where

$$
\mathcal{Q}(b)=a^{2}+a-2-(4 a-1) b+b^{2}
$$


is a quadratic function in terms of $b$. Since the parabola of $\mathcal{Q}(b)$ opens up, it follows from $\mathcal{Q}(1-a)=-6 a(1-a)<0$ and $\mathcal{Q}(1)=-a(3-a)<0$ that $\mathcal{Q}(b)<0$ for $0<a<1$ and $1-a<$ $b<1$. This in conjunction with (2.11) yields $\kappa_{4}(a, b)<\kappa_{3}(a, b)$.

(iii) If the conclusion is not true, that is, $3-3 a-b \leq 0$, it follows that $b \geq \max \{a, 3(1-a)\}$. As we know, $\kappa_{5}(a, b)=b^{2}-2(5 a-1) b+(a+1)^{2}$ is a quadratic function of $b$. We divide the proof into two cases.

CASE 1: $a \geq 3(1-a)$. Then we clearly see that $a \leq b<1$ and $3 / 4 \leq a<1$. Since the symmetric axis $5 a-1>1, \kappa_{5}(a, b)$ is strictly decreasing for $a<b<1$. This gives $\kappa_{5}(a, b) \leq \kappa_{5}(a, a)=-\left[8(a-3 / 4)^{2}+8(a-3 / 4)+1 / 2\right]<0$, which is a contradiction.

CASE 2: $a<3(1-a)$. In other words, $3(1-a)<b<1$ and $2 / 3<a<3 / 4$. Similarly, the monotonicity of $\kappa_{5}(a, b)$ gives rise to $\kappa_{5}(a, b) \leq \kappa_{5}(a, 3(1-a))=4(2 a-1)(5 a-$ $4)<0$, which is also a contradiction.

Lemma 2.7 Let $0<a, b<1$ with $a+b>1$ and $a+b+1 \geq 4 a b$, and $\varphi(r)$ be defined by

$$
\varphi(r)=\frac{1 / r^{\prime a+b-1}-1}{B^{2} /\left(4 r^{\prime a+b+1} \mathcal{K}^{2}\right)-1} .
$$

Then $\varphi(r)$ is strictly decreasing from $(0,1)$ onto $\left(0, \frac{(a+b-1)(a+b+1)}{1+2 a+2 b+a^{2}+b^{2}-6 a b}\right)$.

Proof Let $\varphi_{1}(r)=1 / r^{\prime a+b-1}-1$ and $\varphi_{2}(r)=B^{2} /\left(4 r^{\prime a+b+1} \mathcal{K}^{2}\right)-1$. Then $\varphi(r)=\varphi_{1}(r) / \varphi_{2}(r)$ and $\varphi_{1}(0)=\varphi_{2}(0)=0$. Combining this with Lemma 2.2 , we clearly see that the monotonicity of $\varphi(r)$ depends on $\varphi_{1}^{\prime}(r) / \varphi_{2}^{\prime}(r)$, that is,

$$
\frac{\varphi_{1}^{\prime}(r)}{\varphi_{2}^{\prime}(r)}=\frac{4(a+b-1)}{B^{2}} \cdot\left(r^{\prime} \mathcal{K}\right)^{2} \cdot \frac{r^{2} \mathcal{K}}{(a+1-3 b) r^{2} \mathcal{K}+2(b-a+1)(\mathcal{K}-\mathcal{E})} .
$$

It follows from Lemma 2.3(i) that $(a+1-3 b)+2(b-a+1)(\mathcal{K}-\mathcal{E}) /\left(r^{2} \mathcal{K}\right)$ is strictly increasing from $(0,1)$ onto $\left(\frac{1+2 a+2 b+a^{2}+b^{2}-6 a b}{a+b+1}, 3-a-b\right)$. Since $a+b+1 \geq 4 a b$, Lemma 2.3(iii) leads to the conclusion that $r^{\prime} \mathcal{K}$ is strictly decreasing from $(0,1)$ onto $(0, B / 2)$. This in conjunction with (2.12) implies that $\varphi_{1}^{\prime}(r) / \varphi_{2}^{\prime}(r)$ is strictly decreasing on $(0,1)$.

On the other hand, it follows from L'Hôpital's rule and (2.12) that

$$
\varphi\left(0^{+}\right)=\lim _{r \rightarrow 0^{+}} \frac{\varphi_{1}^{\prime}(r)}{\varphi_{2}^{\prime}(r)}=\frac{(a+b-1)(a+b+1)}{1+2 a+2 b+a^{2}+b^{2}-6 a b}, \quad \varphi\left(1^{-}\right)=0 .
$$

Lemma 2.8 Let $(a, b) \in \Omega_{1}$ and $f(r)$ be defined by

$$
f(r)=\frac{(1-2 b) r^{2} \mathcal{K}+(b-a+1)(\mathcal{K}-\mathcal{E})}{\frac{B^{2}}{4 r^{\prime a+b+1} \mathcal{K}^{2}}-1} .
$$

Then $f(r)$ is strictly decreasing from $(0,1)$ onto $\left(0, \frac{(a+b+1-4 a b) B}{1+2 a+2 b+a^{2}+b^{2}-6 a b}\right)$.

Proof Let $f_{1}(r)=(1-2 b) r^{2} \mathcal{K}+(b-a+1)(\mathcal{K}-\mathcal{E})$ and $f_{2}(r)=B^{2} /\left(4 r^{\prime a+b+1} \mathcal{K}^{2}\right)-1$. Then we clearly see that $f(r)=f_{1}(r) / f_{2}(r)$ and $f_{1}(0)=f_{2}(0)=0$.

By calculations, one has

$$
\frac{f_{1}^{\prime}(r)}{f_{2}^{\prime}(r)}=\frac{4}{B^{2}} \cdot\left[r^{(a+b+1) / 3} \mathcal{K}\right]^{3} \cdot \widehat{f}(r)
$$


where

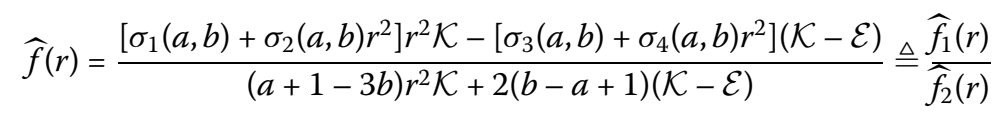

and

$$
\begin{array}{ll}
\sigma_{1}(a, b)=2\left(1-b-a b+b^{2}\right), & \sigma_{2}(a, b)=2(1-b)(2 b-1), \\
\sigma_{3}(a, b)=(a+b-1)(b-a+1), & \sigma_{4}(a, b)=(b-a+1)(3-2 a-2 b) .
\end{array}
$$

Let

$$
\widehat{f}_{11}(r)=\sigma_{1}(a, b)-\sigma_{3}(a, b) \frac{\mathcal{K}-\mathcal{E}}{r^{2} \mathcal{K}}, \quad \widehat{f}_{12}(r)=-r^{2}\left[\sigma_{4}(a, b) \frac{\mathcal{K}-\mathcal{E}}{r^{2} \mathcal{K}}-\sigma_{2}(a, b)\right] .
$$

Then $\widehat{f}_{1}(r) /\left(r^{2} \mathcal{K}\right)=\widehat{f}_{11}(r)+\widehat{f}_{12}(r)$.

It follows from Lemma $2.3(\mathrm{i})$ and $\sigma_{3}(a, b)>0$ that $\widehat{f}_{11}(r)$ is strictly decreasing on $(0,1)$. For $(a, b) \in \Omega_{1}$, namely $0<a, b<1, a+b>1$ and $\kappa_{1}(a, b) \geq 0$, we clearly see from Lemma 2.6(i) that $\kappa_{5}(a, b)=(a+b+1)^{2}-12 a b>0, a+b<3 / 2$, and then $\sigma_{4}(a, b)>0$. This in conjunction with Lemma 2.3(i), (iii) implies that $r^{(a+b+1) / 3} \mathcal{K}$ is strictly decreasing on $(0,1)$ and

$$
\sigma_{4}(a, b) \frac{\mathcal{K}-\mathcal{E}}{r^{2} \mathcal{K}}-\sigma_{2}(a, b)>\sigma_{4}(a, b) \cdot \frac{2 b}{a+b+1}-\sigma_{2}(a, b)=\frac{2 \kappa_{1}(a, b)}{a+b+1} \geq 0 .
$$

Lemma 2.3(i) and (2.15) enable us to know that $\widehat{f}_{12}(r)$ is strictly decreasing on $(0,1)$. This gives the monotonicity of $\widehat{f}_{1}(r) /\left(r^{2} \mathcal{K}\right)$. So $\widehat{f}_{1}(r) /\left(r^{2} \mathcal{K}\right)>\sigma_{1}(a, b)+\sigma_{2}(a, b)-\sigma_{3}(a, b)-$ $\sigma_{4}(a, b)=(2-a-b)(a+b-1)>0$. Moreover, it is easy to verify from Lemma 2.3(i) that $\widehat{f}_{2}(r) /\left(r^{2} \mathcal{K}\right)$ is strictly increasing from $(0,1)$ onto $\left(\frac{1+2 a+2 b+a^{2}+b^{2}-6 a b}{a+b+1}, 3-a-b\right)$. Combining with (2.14), the monotonicity of $\widehat{f}_{1}(r) /\left(r^{2} \mathcal{K}\right)$ and $\widehat{f}_{2}(r) /\left(r^{2} \mathcal{K}\right)$ leads to the conclusion that $\widehat{f}(r)$ is strictly decreasing on $(0,1)$.

Therefore, the monotonicity of $f(r)$ follows from Lemma 2.2 and (2.13) together with the monotonicity of $r^{(a+b+1) / 3} \mathcal{K}$ and $\widehat{f}(r)$.

To this end, by L'Hôpital's rule and (2.13), (2.14),

$$
f\left(0^{+}\right)=\lim _{r \rightarrow 0^{+}} \frac{f_{1}^{\prime}(r)}{f_{2}^{\prime}(r)}=\frac{(a+b+1-4 a b) B}{1+2 a+2 b+a^{2}+b^{2}-6 a b}, \quad f\left(1^{-}\right)=0 .
$$

Lemma 2.9 Let $(a, b) \in \Omega_{2}$ and $g(r)$ be defined by

$$
g(r)=\frac{B^{2} /\left(4 r^{\prime a+b+1} \mathcal{K}^{2}\right)-1}{\left[2 b r^{2} \mathcal{K}+(a-b-1)(\mathcal{K}-\mathcal{E})\right] / r^{\prime 2}} .
$$

Then $g(r)$ is strictly decreasing from $(0,1)$ onto $\left(0, \frac{1+2 a+2 b+a^{2}+b^{2}-6 a b}{4 a b B}\right)$.

Proof Let $g_{1}(r)=B^{2} /\left(4 r^{\prime a+b+1} \mathcal{K}^{2}\right)-1$ and $g_{2}(r)=\left[2 b r^{2} \mathcal{K}+(a-b-1)(\mathcal{K}-\mathcal{E})\right] / r^{\prime 2}$. Then $g(r)=g_{1}(r) / g_{2}(r)$ and $g_{1}(0)=g_{2}(0)=0$.

By calculations, one has

$$
\frac{g_{1}^{\prime}(r)}{g_{2}^{\prime}(r)}=\frac{B^{2}}{4\left[r^{\prime a+b-1} \mathcal{K}\right]^{2}} \cdot \frac{\widehat{g}_{1}(r)}{\widehat{g}_{2}(r)},
$$


where

$$
\begin{aligned}
& \widehat{g}_{1}(r)=\frac{r^{\prime a+b-1}\left[(a-3 b+1) r^{2} \mathcal{K}+2(b-a+1)(\mathcal{K}-\mathcal{E})\right]}{r^{2} \mathcal{K}}, \\
& \widehat{g}_{2}(r)=\frac{\left[\lambda_{1}(a, b)+\lambda_{2}(a, b) r^{2}\right] r^{2} \mathcal{K}+\left[\lambda_{3}(a, b)+\lambda_{4}(a, b) r^{2}\right](\mathcal{K}-\mathcal{E})}{r^{2}}
\end{aligned}
$$

and

$$
\begin{aligned}
& \lambda_{1}(a, b)=2 b(a-b+1), \quad \lambda_{2}(a, b)=4 b^{2}, \\
& \lambda_{3}(a, b)=(b-a+1)(a+b-1), \quad \lambda_{4}(a, b)=-2(a+b)(b-a+1) .
\end{aligned}
$$

By (2.2) and (2.9), we clearly see that

$$
\begin{aligned}
\frac{\mathcal{K}-\mathcal{E}}{r^{2}} & =\frac{B(a, b)}{4(a-1) r} \frac{d F\left(a-1, b ;(a+b+1) / 2 ; r^{2}\right)}{d r} \\
& =\frac{b B(a, b)}{a+b+1} F\left(a, b+1 ;(a+b+3) / 2 ; r^{2}\right) .
\end{aligned}
$$

It follows from (1.2), (1.5), and (2.19) that

$$
\begin{aligned}
& r^{\prime a+b-1} \mathcal{K}=\frac{B(a, b)}{2} F\left(\frac{b-a+1}{2}, \frac{a-b+1}{2} ; \frac{a+b+1}{2} ; r^{2}\right), \\
& r^{\prime a+b-1} \frac{\mathcal{K}-\mathcal{E}}{r^{2}}=\frac{B(a, b) b}{a+b+1} F\left(\frac{b-a+3}{2}, \frac{a-b+1}{2} ; \frac{a+b+3}{2} ; r^{2}\right) .
\end{aligned}
$$

Combining with (2.17), (2.18), (2.20), and (2.21), we rewrite $\widehat{g}_{1}(r)$ and $\widehat{g}_{2}(r)$ in terms of power series:

$$
\begin{aligned}
& \widehat{g}_{1}(r)=\frac{\sum_{n=0}^{\infty} \frac{\left(\frac{a-b+1}{2}, n\right)\left(\frac{b-a+1}{2}, n\right)}{\left(\frac{a+b+1}{2}, n\right) n !} \xi_{a, b}(n) r^{2 n}}{\sum_{n=0}^{\infty} \frac{(a, n)(b, n)}{\left(\frac{a+b+1}{2}, n\right) n !} r^{2 n}}, \\
& \widehat{g}_{2}(r)=\frac{B(a, b)}{2} \sum_{n=0}^{\infty} \frac{(a, n-1)(b, n-1)}{\left(\frac{a+b+1}{2}, n+1\right) n !} \zeta_{a, b}(n) r^{2 n},
\end{aligned}
$$

where

$$
\begin{aligned}
\xi_{a, b}(n)= & \frac{1+2 a+2 b+a^{2}+b^{2}-6 a b+2(a+b+1) n}{1+a+b+2 n}, \\
\zeta_{a, b}(n)= & {\left[(a+b)^{2}-1\right] n^{3}+2(a+b-1)(a+b+2 a b-1) n^{2} } \\
& +\left[4 a b(a+b+a b-1)-3(a+b)^{2}+4(a+b)-1\right] n \\
& +4 a b(1-a)(1-b) .
\end{aligned}
$$

We now claim that $\widehat{g}_{1}(r)$ is strictly decreasing on $(0,1)$ and $\widehat{g}_{2}(r)$ is strictly increasing on $(0,1)$; furthermore, $\widehat{g}_{2}(r)$ has positive Maclaurin coefficients. 
- Lemma 2.1 and (2.22) enable us to know that the monotonicity of $\widehat{g}_{1}(r)$ depends on the monotonicity of the following sequence:

$$
\left\{\alpha_{n}\right\}_{n \geq 0}=\left\{\frac{\left(\frac{a-b+1}{2}, n\right)\left(\frac{b-a+1}{2}, n\right)}{(a, n)(b, n)} \xi_{a, b}(n)\right\}_{n \geq 0} .
$$

A simple calculation yields

$$
\frac{\alpha_{n+1}}{\alpha_{n}}=\frac{(1+b-a+2 n)(1+a-b+2 n) \xi_{a, b}(n+1)}{4(b+n)(a+n) \xi_{a, b}(n)} \leq 1
$$

if and only if

$$
\begin{aligned}
\Delta_{a, b}(n)= & (1+b-a+2 n)(1+a-b+2 n) \xi_{a, b}(n+1) \\
& -4(a+n)(b+n) \xi_{a, b}(n) \\
= & \frac{\widehat{\Delta}_{a, b}(n)}{(1+a+b+2 n)(3+a+b+2 n)} \leq 0,
\end{aligned}
$$

where

$$
\widehat{\Delta}_{a, b}(n)=\kappa_{2}(a, b)+4 \kappa_{3}(a, b) n+4 \kappa_{4}(a, b) n^{2}-16\left[(a+b)^{2}-1\right] n^{3} .
$$

For $(a, b) \in \Omega_{2}$, namely $0<a, b<1, a+b>1, \kappa_{2}(a, b) \leq 0, \kappa_{3}(a, b) \leq 0$, and then $\kappa_{4}(a, b) \leq 0$ by Lemma 2.6(ii). This in conjunction with (2.24) and (2.25) implies that the sequence $\left\{\alpha_{n}\right\}_{n \geq 0}$ is decreasing. So the first assertion is valid.

- We mention that the Pochhammer symbol $(a,-1)(b,-1)=\frac{1}{(a-1)(b-1)}>0$ for $0<a, b<1$. It only needs to prove $\zeta_{a, b}(n)>0$ for $n \geq 0$ with $0<a, b<1$ and $a+b>1$.

Clearly, $\zeta_{a, b}(0)=4 a b(1-a)(1-b)>0$ and $\zeta_{a, b}(1)=4 a b(a+b+2 a b-1)>0$.

Moreover, $\zeta_{a, b}^{\prime}(n)$ is strictly increasing for $n \geq 0$. This gives $\zeta_{a, b}^{\prime}(n) \geq \zeta_{a, b}^{\prime}(1)=4 q(b)$ for $n \geq 1$, where $q(b)=\left(a^{2}+3 a+1\right) b^{2}+\left(3 a^{2}-a-1\right) b+a(a-1)$ is regarded as a quadratic function in terms of $b$ and its parabola opens up.

Observe that

$$
-\frac{3 a^{2}-a-1}{2\left(a^{2}+3 a+1\right)}-(1-a)=-\frac{a(1-a)(2 a+3)+1}{2\left(a^{2}+3 a+1\right)}<0,
$$

that is to say, the symmetric axis of $q(b)$ lies on the left side of the interval $[1-a, 1]$.

This in conjunction with $q(1-a)=a^{2}(a-1)^{2}>0$ implies that $q(b)>0$ for $1-a<b<1$. So $\zeta_{a, b}(n)$ is strictly increasing for $n \geq 1$ and $\zeta_{a, b}(n) \geq \zeta_{a, b}(1)>0$ for $n \geq 1$. This completes the second assertion.

Therefore, $\widehat{g}_{1}(r) / \widehat{g}_{2}(r)$ is strictly decreasing on $(0,1)$ follows from the above assertions together with $\widehat{g}_{1}(r)>0$ and $\widehat{g}_{2}(r)>0$. Combining this with (2.16), Lemma 2.2 and Lemma 2.3(ii), we conclude that $g(r)$ is strictly decreasing on $(0,1)$.

It remains to compute two end values of $g(r)$. By L'Hôpital's rule and (2.16) together with Lemma 2.3(i), (ii),

$$
g\left(0^{+}\right)=\lim _{r \rightarrow 0^{+}} \frac{g_{1}^{\prime}(r)}{g_{2}^{\prime}(r)}=\frac{1+2 a+2 b+a^{2}+b^{2}-6 a b}{4 a b B}, \quad g\left(1^{-}\right)=0 .
$$


Lemma 2.10 Let $0<a \leq b<1$ with $a+b>1$ and $\kappa_{5}(a, b) \geq 0$, and $h(r)$ be defined by

$$
h(r)=\frac{2 b r^{2} \mathcal{K}-\left[2(a+b-1)+(3-3 a-b) r^{2}\right](\mathcal{K}-\mathcal{E})}{(a-3 b+1) r^{2} \mathcal{K}+2(b-a+1)(\mathcal{K}-\mathcal{E})} .
$$

Then $h(r)$ is strictly decreasing from $(0,1)$ onto $\left(\frac{a+b-1}{3-a-b}, \frac{2 b(3-a-b)}{1+2 a+2 b+a^{2}+b^{2}-6 a b}\right)$.

Proof We denote by $h_{1}(r)=2 b-\left[2(a+b-1)+(3-3 a-b) r^{2}\right](\mathcal{K}-\mathcal{E}) /\left(r^{2} \mathcal{K}\right)$ and $h_{2}(r)=$ $(a-3 b+1)+2(b-a+1)(\mathcal{K}-\mathcal{E}) /\left(r^{2} \mathcal{K}\right)$.

If $0<a \leq b<1, a+b>1$, and $\kappa_{5}(a, b) \geq 0$, then $3-3 a-b>0$ follows from Lemma 2.6(iii). Combining this with Lemma 2.3(i), we conclude that $h_{1}(r)$ is strictly decreasing from $(0,1)$ onto $\left(a+b-1, \frac{2 b(3-a-b)}{a+b+1}\right)$ and $h_{2}(r)$ is strictly increasing from $(0,1)$ onto $\left(\frac{1+2 a+2 b+a^{2}+b^{2}-6 a b}{a+b+1}, 3-\right.$ $a-b)$. This gives the monotonicity of $h(r)=h_{1}(r) / h_{2}(r)$ together with two limiting values $h\left(0^{+}\right)$and $h\left(1^{-}\right)$.

\section{Main results}

Theorem 3.1 Let $(a, b) \in \Omega_{1}$ and $F(r)$ be defined on $(0,1)$ by

$$
F(r)=\frac{D r^{1-a-b}+E-m_{a, b}(r)}{D r^{1-a-b}+E-\mu_{a, b}(r)}
$$

Then $F(r)$ is strictly decreasing from $(0,1)$ onto $\left(1, L_{0}\right)$, where

$$
L_{0}=\frac{(a+b+1)\left[(a+b-1)^{2}+4\right]-16 a b}{(a+b-1)\left(1+2 a+2 b+a^{2}+b^{2}-6 a b\right)} .
$$

In particular, the double inequality

$$
m_{a, b}(r)<\mu_{a, b}(r)<\frac{1}{L_{0}} m_{a, b}(r)+\left(1-\frac{1}{L_{0}}\right)\left(D r^{1-a-b}+E\right)
$$

holds for $r \in(0,1)$.

Proof Let $F_{1}(r)=D r^{1-a-b}+E-m_{a, b}(r)$ and $F_{2}(r)=D r^{1-a-b}+E-\mu_{a, b}(r)$. Clearly, $F(r)=$ $F_{1}(r) / F_{2}(r)$ and $F_{1}\left(0^{+}\right)=F_{2}\left(0^{+}\right)=0$ follow from Corollary 2.5 .

By calculations, one has

$$
\begin{aligned}
\frac{F_{1}^{\prime}(r)}{F_{2}^{\prime}(r)} & =\frac{\left(\frac{1}{r^{\prime a+b-1}}-1\right)+\frac{4 r^{a+b-1} \mathcal{K}^{\prime}}{(a+b-1) B D}\left[(1-2 b) r^{2} \mathcal{K}+(b-a+1)(\mathcal{K}-\mathcal{E})\right]}{\frac{B^{2}}{4 r^{\prime a+b+1} \mathcal{K}^{2}}-1} \\
& =\varphi(r)+\frac{4}{(a+b-1) B D} \cdot r^{a+b-1} \mathcal{K}^{\prime} \cdot f(r)
\end{aligned}
$$

where $\varphi(r)$ and $f(r)$ are defined as in Lemma 2.7 and Lemma 2.8, respectively.

Since $r^{a+b-1} \mathcal{K}^{\prime}$ can be regarded as the composition of $x^{\prime a+b-1} \mathcal{K}(x)$ and $x=r^{\prime}=\sqrt{1-r^{2}}$, Lemma 2.3(ii) enables us to know that $r^{a+b-1} \mathcal{K}^{\prime}$ is strictly decreasing from $(0,1)$ onto $(B / 2, D)$. This in conjunction with (3.1) together with Lemma 2.2, Lemma 2.7, and Lemma 2.8 gives rise to the monotonicity of $F(r)$ and also, by L'Hôpital's rule 
and (3.1),

$$
F\left(0^{+}\right)=\lim _{r \rightarrow 0^{+}} \frac{F_{1}^{\prime}(r)}{F_{2}^{\prime}(r)}=\varphi\left(0^{+}\right)+\frac{4}{(a+b-1) B D} \cdot D \cdot f\left(0^{+}\right)=L_{0},
$$

and $F\left(1^{-}\right)=1$ follows directly from $m_{a, b}\left(1^{-}\right)=\mu_{a, b}\left(1^{-}\right)=0$.

Corollary 3.2 Let $(a, b) \in \Omega_{1}$ and $\widehat{F}(r)$ be defined on $(0,1)$ by

$$
\widehat{F}(r)=\frac{m_{a, b}(r)-D\left(r^{1-a-b}-1\right)}{\mu_{a, b}(r)-D\left(r^{1-a-b}-1\right)} .
$$

Then $\widehat{F}(r)$ is strictly decreasing from $(0,1)$ onto $(0,1)$.

Proof Let $\widehat{F}_{1}(r)=m_{a, b}(r)-D\left(r^{1-a-b}-1\right)$ and $\widehat{F}_{2}(r)=\mu_{a, b}(r)-D\left(r^{1-a-b}-1\right)$. Then $\widehat{F}(r)=$ $\widehat{F}_{1}(r) / \widehat{F}_{2}(r)$ and $\widehat{F}_{1}\left(1^{-}\right)=\widehat{F}_{2}\left(1^{-}\right)=0$.

Since $\widehat{F}_{1}^{\prime}(r) / \widehat{F}_{2}^{\prime}(r)=F_{1}^{\prime}(r) / F_{2}^{\prime}(r)$, Lemma 2.2 enables us to know the monotonicity of $\widehat{F}(r)$ depends on that of $F_{1}^{\prime}(r) / F_{2}^{\prime}(r)$, which follows from Theorem 3.1. It only remains to compute two limiting values $\widehat{F}\left(0^{+}\right)$and $\widehat{F}\left(1^{-}\right)$.

By Corollary 2.5, it is easy to see that $\widehat{F}\left(0^{+}\right)=(D+E) /(D+E)=1$. By L'Hôpital's rule and (3.1) together with Lemma 2.7, Lemma 2.8,

$$
\widehat{F}\left(1^{-}\right)=\lim _{r \rightarrow 1^{-}} \frac{\widehat{F}_{1}^{\prime}(r)}{\widehat{F}_{2}^{\prime}(r)}=\varphi\left(1^{-}\right)+\frac{4}{(a+b-1) B D} \cdot \frac{B}{2} \cdot f\left(1^{-}\right)=0 .
$$

Theorem 3.3 Let $(a, b) \in \Omega_{2}$ and $G(r)$ be defined on $(0,1)$ by

$$
G(r)=\frac{D r^{1-a-b}+E-\mu_{a, b}(r)}{\mathcal{K}-B / 2} .
$$

Then $G(r)$ is strictly decreasing from $(0,1)$ onto $(0, \infty)$.

Proof We denote $G_{1}(r)=D r^{1-a-b}+E-\mu_{a, b}(r)$ and $G_{2}(r)=\mathcal{K}-B / 2$. Then we clearly see that $G(r)=G_{1}(r) / G_{2}(r)$ and $G_{1}\left(0^{+}\right)=G_{2}\left(0^{+}\right)=0$.

By taking the derivative of $G_{1}(r)$ and $G_{2}(r)$, one has

$$
\begin{aligned}
\frac{G_{1}^{\prime}(r)}{G_{2}^{\prime}(r)} & =\frac{(a+b-1) D}{r^{a+b-1}} \cdot \frac{B^{2} /\left(4 r^{\prime a+b+1} \mathcal{K}^{2}\right)-1}{\left[2 b r^{2} \mathcal{K}+(a-b-1)(\mathcal{K}-\mathcal{E})\right] / r^{\prime 2}} \\
& =\frac{(a+b-1) D}{r^{a+b-1}} \cdot g(r),
\end{aligned}
$$

where $g(r)$ is defined as in Lemma 2.9.

Therefore, the monotonicity of $G(r)$ follows from Lemma 2.9 and that of $1 / r^{a+b-1}$.

To this end, by L'Hôpital's rule and (3.2),

$$
G\left(0^{+}\right)=\lim _{r \rightarrow 0^{+}} \frac{G_{1}^{\prime}(r)}{G_{2}^{\prime}(r)}=\lim _{r \rightarrow 0^{+}} \frac{(a+b-1) D}{r^{a+b-1}} \cdot g\left(0^{+}\right)=\infty, \quad G\left(1^{-}\right)=0 .
$$

Theorem 3.4 Let $(a, b) \in \Omega_{3}$ and $H(r)$ be defined on $(0,1)$ by

$$
H(r)=\frac{r^{1-a-b}(B / 2-\mathcal{E})}{D r^{1-a-b}+E-\mu_{a, b}(r)} .
$$


Then $H(r)$ is strictly decreasing from $(0,1)$ onto $\left(L_{1}, L_{2}\right)$, where

$$
\begin{aligned}
L_{1} & =\frac{2 b(1-a)(3-a-b) B}{(a+b-1)\left(1+2 a+2 b+a^{2}+b^{2}-6 a b\right) D}, \\
L_{2} & =\frac{(b-a+1) B+2(a+b-1) E}{2(b-a+1)(D+E)} .
\end{aligned}
$$

As a consequence, the double inequality

$$
r^{1-a-b}\left(D-\frac{B}{2 L_{1}}+\mathcal{E}\right)+E<\mu_{a, b}(r)<r^{1-a-b}\left(D-\frac{B}{2 L_{2}}+\mathcal{E}\right)+E
$$

holds for $r \in(0,1)$.

Proof Since $H(r)$ is symmetric with respect to $a, b$, we may assume that $0<a \leq b<1$. Let $H_{1}(r)=r^{1-a-b}(B / 2-\mathcal{E})$ and $H_{2}(r)=D r^{1-a-b}+E-\mu_{a, b}(r)$. Then we clearly see from Corollary 2.5 and $a+b<2$ that $H_{1}(r)=H_{1}(r) / H_{2}(r)$ and $H_{1}\left(0^{+}\right)=H_{2}\left(0^{+}\right)=0$.

Moreover,

$$
\begin{aligned}
\frac{H_{1}^{\prime}(r)}{H_{2}^{\prime}(r)} & =\frac{r^{-(a+b)}[(1-a-b) B / 2+(3 a+b-3) \mathcal{E}+2(1-a) \mathcal{K}]}{(a+b-1) D r^{-(a+b)}\left(\frac{B^{2}}{4 r^{\prime a+b+1} \mathcal{K}^{2}}-1\right)} \\
& =\frac{1}{(a+b-1) D} \cdot \frac{(1-a-b) B / 2+(3 a+b-3) \mathcal{E}+2(1-a) \mathcal{K}}{\left(\frac{B^{2}}{4 r^{(a+b+1} \mathcal{K}^{2}}-1\right)} \\
& \triangleq \frac{H_{11}(r)}{H_{22}(r)} \\
H_{11}\left(0^{+}\right) & =H_{22}\left(0^{+}\right)=0,
\end{aligned}
$$

and

$$
\frac{H_{11}^{\prime}(r)}{H_{22}^{\prime}(r)}=\frac{8(1-a)}{(a+b-1) B^{2} D} \cdot\left(r^{\prime} \frac{a+b+1}{3} \mathcal{K}\right)^{3} \cdot h(r),
$$

where $h(r)$ is defined as in Lemma 2.10 .

If $(a, b) \in \Omega_{3}$, in other words, $0<a \leq b<1, a+b>1$, and $\kappa_{5}(a, b)=(a+b+1)^{2}-12 a b \geq 0$, then it follows from Lemma 2.3(iii) and Lemma 2.10 that $r^{\prime \frac{a+b+1}{3}} \mathcal{K}$ is strictly decreasing on $(0,1)$ and $h(r)$ is strictly decreasing on $(0,1)$. This in conjunction with (3.3), (3.4), and Lemma 2.2 implies that $H(r)$ is strictly decreasing on $(0,1)$. By L'Hôpital's rule together with Lemma 2.10 and (3.3), (3.4),

$$
\begin{aligned}
H\left(0^{+}\right) & =\lim _{r \rightarrow 0^{+}} H(r)=\lim _{r \rightarrow 0^{+}} \frac{H_{1}^{\prime}(r)}{H_{2}^{\prime}(r)}=\lim _{r \rightarrow 0^{+}} \frac{H_{11}^{\prime}(r)}{H_{22}^{\prime}(r)} \\
& =\frac{8(1-a)}{(a+b-1) B^{2} D} \cdot\left(\frac{B}{2}\right)^{3} \cdot h\left(0^{+}\right)=L_{1},
\end{aligned}
$$

and $H\left(1^{-}\right)=L_{2}$ follows easily from $\mu_{a, b}\left(1^{-}\right)=0$.

Open Problem What is the sub-region of $\left\{(a, b) \in \mathbb{R}^{2} \mid 0<a, b<1\right\}$ such that the function

$$
\widehat{G}(r)=\frac{D r^{1-a-b}+E-\mu_{a, b}(r)}{r^{1-a-b}(\mathcal{K}-B / 2)}
$$


is strictly decreasing from $(0,1)$ onto $\left(0, L_{3}\right)$, where

$$
L_{3}=\frac{(a+b-1)\left(1+2 a+2 b+a^{2}+b^{2}-6 a b\right) D}{2 a b(3-a-b) B} .
$$

\section{Consequences and discussion}

In the article, we study the monotonicity of the functions $F(r), G(r)$, and $H(r)$ related to generalized Grötzsch ring function and generalized elliptic integrals, where $F(r), G(r)$, and $H(r)$ are explicitly given by

$$
F(r)=\frac{D r^{1-a-b}+E-m_{a, b}(r)}{D r^{1-a-b}+E-\mu_{a, b}(r)}, \quad G(r)=\frac{D r^{1-a-b}+E-\mu_{a, b}(r)}{\mathcal{K}-B / 2},
$$

and

$$
H(r)=\frac{r^{1-a-b}(B / 2-\mathcal{E})}{D r^{1-a-b}+E-\mu_{a, b}(r)} .
$$

\section{Conclusion}

In the article, we have found the sub-regions of $\left\{(a, b) \in \mathbb{R}^{2} \mid 0<a, b<1, a+b>1\right\}$ such that several quotient functions involving $\mu_{a, b}(r), \mathcal{K}_{a, b}(r), \mathcal{E}_{a, b}(r)$, and $m_{a, b}(r)$ are monotonic on their corresponding sub-regions, and established several inequalities for $\mu_{a, b}(r)$ and $m_{a, b}(r)$. Our results are the variants and extensions of the previous results of [42, Theorems 1.1 and 1.2] in the case of zero-balanced.

\section{Acknowledgements}

The authors would like to express their sincere thanks to the editor and the anonymous reviewers for their helpful comments and suggestions.

\section{Funding}

This work was supported by the National Natural Science Foundation of China (Grant Nos. 11971142, 11871202) and the Natural Science Foundation of Zhejiang Province (Grant No. LY19A010012).

Availability of data and materials

Not applicable.

\section{Competing interests}

The authors declare that they have no competing interests.

\section{Authors' contributions}

All authors contributed equally to the writing of this paper. All authors read and approved the final manuscript.

\section{Publisher's Note}

Springer Nature remains neutral with regard to jurisdictional claims in published maps and institutional affiliations.

Received: 25 July 2019 Accepted: 28 February 2020 Published online: 11 March 2020

\section{References}

1. Abramowitz, M., Stegun, I.A.: Handbook of Mathematical Functions with Formulas, Graphs, and Mathematical Tables. U.S. Govt. Printing Office, Washington (1964)

2. Li, Y.-Q., Wang, S.-X., Zhou, X.-W., Zhu, N.: Diffusion occupation time before exiting. Front. Math. China 9(4), 843-862 (2014)

3. Wang, M.-K., Chu, Y.-M., Song, Y.-Q.: Asymptotical formulas for Gaussian and generalized hypergeometric functions. Appl. Math. Comput. 276, 44-60 (2016)

4. Wang, M.-K., Chu, Y.-M., Jiang, Y.-P.: Ramanujan's cubic transformation inequalities for zero-balanced hypergeometric functions. Rocky Mt. J. Math. 46(2), 679-691 (2016)

5. Wang, M.-K., Chu, Y.-M.:- Refinements of transformation inequalities for zero-balanced hypergeometric functions. Acta Math. Sci. 37B(3), 607-622 (2017) 
6. Qian, W.-M., Zhang, W., Chu, Y.-M.: Bounding the convex combination of arithmetic and integral mean in terms of one-parameter harmonic and geometric means. Miskolc Math. Notes 20(2), 1157-1166 (2019)

7. Wang, M.-K., Chu, Y.-M.: Landen inequalities for a class of hypergeometric functions with applications. Math. Inequal. Appl. 21(2), 521-537 (2018)

8. Zhao, T.-H., Wang, M.-K., Zhang, W., Chu, Y.-M.: Quadratic transformation inequalities for Gaussian hypergeometric function. J. Inequal. Appl. 2018, Article ID 251 (2018)

9. Wang, J.-F., Chen, X.-Y., Huang, L.-H.: The number and stability of limit cycles for planar piecewise linear systems of node-saddle type. J. Math. Anal. Appl. 469(1), 405-427 (2019)

10. Hu, X.-M., Tian, J.-F., Chu, Y.-M., Lu, Y.-X.: On Cauchy-Schwarz inequality for $\mathrm{N}$-tuple diamond-alpha integral. J. Inequal. Appl. 2020, Article ID 8 (2020)

11. Qiu, S.-L., Ma, X.-Y., Chu, Y.-M.: Sharp Landen transformation inequalities for hypergeometric functions, with applications. J. Math. Anal. Appl. 474(2), 1306-1337 (2019)

12. Wang, M.-K., Chu, Y.-M., Zhang, W.: Monotonicity and inequalities involving zero-balanced hypergeometric function. Math. Inequal. Appl. 22(2), 601-617 (2019)

13. Wang, M.-K., Chu, Y.-M., Zhang, W.: Precise estimates for the solution of Ramanujan's generalized modular equation. Ramanujan J. 49(3), 653-668 (2019)

14. Wang, M.-K., Zhang, W., Chu, Y.-M.: Monotonicity, convexity and inequalities involving the generalized elliptic integrals. Acta Math. Sci. 39B(5), 1440-1450 (2019)

15. Wang, M.-K., Chu, H.-H., Chu, Y.-M.: Precise bounds for the weighted Hölder mean of the complete $p$-elliptic integrals. J. Math. Anal. Appl. 480(2), Article ID 123388 (2019). https://doi.org/10.1016/j.jmaa.2019.123388

16. Wang, M.-K., He, Z.-Y., Chu, Y.-M.: Sharp power mean inequalities for the generalized elliptic integral of the first kind. Comput. Methods Funct. Theory 20, 111-124 (2020). https://doi.org/10.1007/s40315-020-00298-w

17. Yang, Z.-H., Qian, W.-M., Zhang, W., Chu, Y.-M.: Notes on the complete elliptic integral of the first kind. Math. Inequal. Appl. 23(1), 77-93 (2020)

18. Qian, W.-M., He, Z.-Y., Chu, Y.-M.: Approximation for the complete elliptic integral of the first kind. Rev. R. Acad. Cienc. Exactas Fís. Nat., Ser. A Mat. 114, 57 (2020). https://doi.org/10.1007/s13398-020-00784-9

19. Anderson, G.D., Vamanamurthy, M.K., Vuorinen, M.: Conformal Invariants, Inequalities, and Quasiconformal Maps. Wiley, New York (1997)

20. Qi, F.: Bounds for the ratio of two gamma functions. J. Inequal. Appl. 2010, Article ID 493058 (2010)

21. Guo, B.-N., Qi, F.: An extension of an inequality for ratios of gamma functions. J. Approx. Theory 163(9), 1208-1216 (2011)

22. Zhao, T.-H., Chu, Y.-M., Wang, H.: Logarithmically complete monotonicity properties relating to the gamma function. Abstr. Appl. Anal. 2011, Article ID 896483 (2011)

23. Latif, M.A., Rashid, S., Dragomir, S.S., Chu, Y.-M.: Hermite-Hadamard type inequalities for co-ordinated convex and quasi-convex functions and their applications. J. Inequal. Appl. 2019, Article ID 317 (2019)

24. Qi, F: Integral representations and complete monotonicity related to the remainder of Burnside's formula for the gamma function. J. Comput. Appl. Math. 268, 155-167 (2014)

25. Iqbal, A., Adil Khan, M., Ullah, S., Chu, Y.-M.: Some Hermite-Hadamard type integral inequalities associated with conformable fractional integrals and their application. J. Funct. Spaces 2020, Article ID 9845407 (2020)

26. Yang, Z.-H., Qian, W.-M., Chu, Y.-M., Zhang, W.: On rational bounds for the gamma function. J. Inequal. Appl. 2017, Article ID 210 (2017)

27. Alzer, H.: Inequalities for the beta function. Anal. Math. 40(1), 1-11 (2014)

28. Huang, C.-X., Yang, Z.-C., Yi, T.-S., Zou, X.-F.: On the basins of attraction for a class of delay differential equations with non-monotone bistable nonlinearities. J. Differ. Equ. 256(7), 2101-2114 (2014)

29. Wang, J.-F., Huang, C.-X., Huang, L.-H.: Discontinuity-induced limit cycles in a general planar piecewise linear system of saddle-focus type. Nonlinear Anal. Hybrid Syst. 33, 162-178 (2019)

30. Huang, C.-X., Zhang, H., Huang, L.-H.: Almost periodicity analysis for a delayed Nicholson's blowflies model with nonlinear density-dependent mortality term. Commun. Pure Appl. Anal. 18(6), 3337-3349 (2019)

31. Huang, T.-R., Han, B.-W., Ma, X.-Y., Chu, Y.-M.: Optimal bounds for the generalized Euler-Mascheroni constant. J. Inequal. Appl. 2018, Article ID 118 (2018)

32. Tian, Z.-L., Liu, Y., Zhang, Y., Liu, Z.-Y., Tian, M.-Y.: The general inner-outer iteration method based on regular splittings for the PageRank problem. Appl. Math. Comput. 356, 479-501 (2019)

33. $\mathrm{Hu}, \mathrm{H} .-$-., Yi, T.-S., Zou, X.-F.: On spatial-temporal dynamics of a Fisher-KPP equation with a shifting environment. Proc Am. Math. Soc. 148(1), 213-221 (2020)

34. Heikkala, V., Vamanamurthy, M.K., Vuorinen, M.: Generalized elliptic integrals. Comput. Methods Funct. Theory 9(1), 75-109 (2009)

35. Heikkala, V., Lindén, H., Vamanamurthy, M.K., Vuorinen, M.: Generalized elliptic integrals and the Legendre $\mathcal{M}$-function. J. Math. Anal. Appl. 338(1), 223-243 (2008)

36. Wang, M.-K., Qiu, S.-L., Chu, Y.-M., Jiang, Y.-P.: Generalized Hersch-Pfluger distortion function and complete elliptic integrals. J. Math. Anal. Appl. 385(1), 221-229 (2012)

37. Khan, S., Adil Khan, M., Chu, Y.-M.: Converse of the Jensen inequality derived from the Green functions with applications in information theory. Math. Methods Appl. Sci. 43(5), 2577-2587 (2020)

38. Bhayo, B.A., Vuorinen, M.: On generalized complete elliptic integrals and modular functions. Proc. Edinb. Math. Soc. (2) 55(3), 591-611 (2012)

39. Qiu, S.-L., Qiu, Y.-F., Wang, M.-K., Chu, Y.-M.: Hölder mean inequalities for the generalized Grötzsch ring and Hersch-Pfluger distortion functions. Math. Inequal. Appl. 15(1), 237-245 (2012)

40. Ma, X.-Y., Wang, M.-K., Zhong, G.-H., Qiu, S.-L., Chu, Y.-M.: Some inequalities for the generalized distortion functions, Math. Inequal. Appl. 15(4), 941-954 (2012)

41. Takeuchi, S.: A new form of the generalized complete elliptic integrals. Kodai Math. J. 39(1), 202-226 (2016)

42. Ma, X.-Y., Qiu, S.-L., Tu, G.-Y.: Generalized Grötzsch ring function and generalized elliptic integrals. Appl. Math. J. Chin. Univ. 31B(4), 458-468 (2016)

43. Kamiya, T., Takeuchi, S.: Complete $(p, q)$-elliptic integrals with application to a family of means. J. Class. Anal. 10(1), $15-25(2017)$ 
44. Yang, Z.-H., Chu, Y.-M.: A monotonicity property involving the generalized elliptic integral of the first kind. Math. Inequal. Appl. 20(3), 729-735 (2017)

45. Wang, M.-K., Li, Y.-M., Chu, Y.-M.: Inequalities and infinite product formula for Ramanujan generalized modular equation function. Ramanujan J. 46(1), 189-200 (2018)

46. Huang, T.-R., Tan, S.-Y., Ma, X.-Y., Chu, Y.-M.: Monotonicity properties and bounds for the complete $p$-elliptic integrals J. Inequal. Appl. 2018, 239 (2018)

47. Zhao, T.-H., Shi, L., Chu, Y.-M.: Convexity and concavity of the modified Bessel functions of the first kind with respect to Hölder means. Rev. R. Acad. Cienc. Exactas Fís. Nat., Ser. A Mat. https://doi.org/10.1007/s13398-020-00825-3

48. Abbas Baloch, I., Chu, Y.-M.: Petrović-type inequalities for harmonic h-convex functions. J. Funct. Spaces 2020, Article ID $3075390(2020)$

49. Wang, F., He, J.-H., Yin, L., Qi, F.: Monotonicity properties and inequalities related to generalized Grötzsch ring functions. Open Math. 17(1), 802-812 (2019)

50. Adil Khan, M., Mohammad, N., Nwaeze, E.R., Chu, Y.-M.: Quantum Hermite-Hadamard inequality by means of Green function. Adv. Differ. Equ. 2020 (2020). https://doi.org/10.1186/s13662-020-02559-3

51. Wang, M.-K., Chu, Y.-M., Qiu, Y.-F., Qiu, S.-L.: An optimal power mean inequality for the complete elliptic integrals. Appl. Math. Lett. 24(6), 887-890 (2011)

52. Lin, L., Liu, Z.-Y.: An alternating projected gradient algorithm for nonnegative matrix factorization. Appl. Math. Comput. 217(24), 9997-10002 (2011)

53. Zhang, L., Li, J.-L.: A new globalization technique for nonlinear conjugate gradient methods for nonconvex minimization. Appl. Math. Comput. 217(24), 10295-10304 (2011)

54. Chu, Y.-M., Wang, M.-K., Qiu, S.-L.: Optimal combinations bounds of root-square and arithmetic means for Toader mean. Proc. Indian Acad. Sci. Math. Sci. 122(1), 41-51 (2012)

55. Zhou, W.-J.: On the convergence of the modified Levenberg-Marquardt method with a nonmonotone second order Armijo type line search. J. Comput. Appl. Math. 239, 152-161 (2013)

56. Zhang, L., Jian, S.-Y.: Shuyuan further studies on the Wei-Yao-Liu nonlinear conjugate gradient method. Appl. Math. Comput. 219(14), 7616-7621 (2013)

57. Zhao, J., Liu, J.-B., Fang, L.-J.: Anti-periodic boundary value problems of second-order functional differential equations Bull. Malays. Math. Sci. Soc. 37(2), 311-320 (2014)

58. Zhou, X.-S.: Weighted sharp function estimate and boundedness for commutator associated with singular integral operator satisfying a variant of Hörmander's condition. J. Math. Inequal. 9(2), 587-596 (2015)

59. Fang, X.-P., Deng, Y.-J., Li, J.: Plasmon resonance and heat generation in nanostructures. Math. Methods Appl. Sci. 38(18), 4663-4672 (2015)

60. Wang, W.-S.: On A-stable one-leg methods for solving nonlinear Volterra functional differential equations. Appl. Math Comput. 314, 380-390 (2017)

61. Liu, Z.-Y., Wu, N.-C., Qin, X.-R., Zhang, Y.-L.: Trigonometric transform splitting methods for real symmetric Toeplitz systems. Comput. Math. Appl. 75(8), 2782-2794 (2018)

62. Zhu, K.-X., Xie, Y.-Q., Zhou, F.: Pullback attractors for a damped semilinear wave equation with delays. Acta Math. Sin. 34(7), 1131-1150 (2018)

63. Yang, Z.-H., Chu, Y.-M., Zhang, W.: High accuracy asymptotic bounds for the complete elliptic integral of the second kind. Appl. Math. Comput. 348, 552-564 (2019)

64. Qian, W.-M., He, Z.-Y., Zhang, H.-W., Chu, Y.-M.: Sharp bounds for Neumann means in terms of two-parameter contraharmonic and arithmetic mean. J. Inequal. Appl. 2019, Article ID 168 (2019)

65. Qian, W.-M., Yang, Y.-Y., Zhang, H.-W., Chu, Y.-M.: Optimal two-parameter geometric and arithmetic mean bounds for the Sándor-Yang mean. J. Inequal. Appl. 2019, Article ID 287 (2019)

66. Zaheer Ullah, S., Adil Khan, M., Chu, Y.-M.: A note on generalized convex functions. J. Inequal. Appl. 2019, Article ID $291(2019)$

67. Wang, M.-K., Hong, M.-Y., Xu, Y.-F., Shen, Z.-H., Chu, Y.-M.: Inequalities for generalized trigonometric and hyperbolic functions with one parameter. J. Math. Inequal. 14(1), 1-21 (2020)

68. Rafeeq, S., Kalsoom, H., Hussain, S., Rashid, S., Chu, Y.-M.: Delay dynamic double integral inequalities on time scales with applications. Adv. Differ. Equ. 2020, Article ID 40 (2020)

69. Wang, B., Luo, C.-L., Li, S.-H., Chu, Y.-M.: Sharp one-parameter geometric and quadratic means bounds for the Sándor-Yang means. Rev. R. Acad. Cienc. Exactas Fís. Nat., Ser. A Mat. 114, 7 (2020) https://doi.org/10.1007/s13398-019-00734-0

70. Yang, Z.-H., Chu, Y.-M., Wang, M.-K.: Monotonicity criterion for the quotient of power series with applications. J. Math. Anal. Appl. 428(1), 587-604 (2015) 